\title{
Photon yield enhancement of red fluorophores at cryogenic temperatures
}

\author{
Christiaan N. Hulleman ${ }^{* \dagger} \quad$ Weixing Li $\ddagger \S \quad$ Ingo Gregor ${ }^{\ddagger}$ \\ Bernd Rieger ${ }^{\dagger} \quad$ Jörg Enderlein ${ }^{\ddagger}$
}

\begin{abstract}
Single Molecule Localization Microscopy has become one of the most successful and widely applied methods of Super-resolution Fluorescence Microscopy. Its achievable resolution strongly depends on the number of detectable photons from a single molecule until photobleaching. By cooling a sample from room temperature down to liquid nitrogen temperatures, the photostability of dyes can be enhanced by more than 100 fold, which results in an improvement in localization precision greater than 10 times. Here, we investigate a variety of fluorescent dyes in the red spectral region, and we find an average photon yield between $3.5 \cdot 10^{6}$ to $11 \cdot 10^{6}$ photons before bleaching at liquid nitrogen temperatures, corresponding to a theoretical localization precision around $0.1 \mathrm{~nm}$.
\end{abstract}

\section{Introduction}

The last 25 years have seen a revolution in optical far field microscopy, in particular due to the invention of super-resolution methods such as STED, PALM and STORM [1]. Especially the two latter methods, which are methods of the class of Single-Molecule Localization Microscopy (SMLM), now routinely achieve image resolutions of $10-20 \mathrm{~nm}[2,3]$. The attainable localization precision of SMLM is ultimately limited by the amount of detected photons until bleaching (or photoswitching), and follows the generic law

$$
\sigma_{l o c}=\frac{\sigma_{p s f}}{\sqrt{N}} \approx \frac{0.25 \lambda}{\mathrm{NA} \sqrt{N}},
$$

where $\lambda$ is the emission wavelength, NA the numerical aperture of the objective, and $N$ is the number of detected photons [4]. More complex equations have

\footnotetext{
*corresponding author: c.n.hulleman@tudelft.nl

${ }^{\dagger}$ Quantitative Imaging Group, Delft University of Technology, Lorentzweg 1, 2628CJ Delft (The Netherlands)

${ }^{\ddagger}$ Georg-August-Universität Göttingen, III. Physical Institute Biophysics / Complex Systems, Friedrich-Hund-Platz 1, 37077 Göttingen (Germany)

$\S$ Current Address: Institute of Biophysics, Chinese Academy of Sciences, Datun Road 15, 100101, Beijing, China
} 
been developed taking background and camera pixelation into account [5], but equation 1 represents the most favorable scenario and thus a lower limit for the attainable resolution.

As can be seen, one way to increase the achievable localization accuracy and thus image resolution is to increase the NA. However, another way is to increase the number of detected photons $N$. Thus, by cooling a sample and thereby increasing the photostability of the fluorescent dye molecules can have a tremendous impact on resolution in SMLM. Cooling reduces all photochemical reaction rates and thus inhibits bleaching, thereby enabling significantly more photons to be detected from a molecule, resulting in a localization precision in the Ångström range [6, 7]. Weisenburger et al. compared the performance of dyes with an emission peak of $552-576 \mathrm{~nm}$ at liquid helium temperatures (4.4 $\mathrm{K}$ ), finding an increase of one to two orders of magnitude in the number of emitted photons [6]. Li et al. built a cryo-fluorescence microscope with samples contained in a liquid nitrogen cryostat and analyzed the performance of ATTO $647 \mathrm{~N}$, finding an increase of more than two orders of magnitude in photon yield [7]. One more advantage of cryo-fluorescence microscopy is that it offers the possibility for correlative light and electron microscopy (cryo-CLEM), adding the specificity of fluorescent labeling to the superior structural resolution of electron microscopy (cryo-EM) $[8,9]$.

The resolution in a super-resolution image is not solely determined by the localization accuracy but equally by the density of emitters [10]. In cases where one can image many instances of a biological structure, the synthesis of many of these images into one final super-super-resolution image (particle averaging) can tremendously increase the effective label density and signal-to-noise ratio. In this case, the achievable resolution is finally only determined by the singlemolecule localization precision $\sigma_{l o c}[11,12]$. Here, we see the biggest possible impact that improved localization precision at cryogenic temperatures can have on resolving molecular details with Ångström resolution.

For imaging of biological samples, red fluorescent dyes are particularly interesting due to the reduced auto-fluorescence and lower scattering intensity in the red spectral region [13]. Wurm et al. investigated the performance of red fluorophores at room temperature, comparing ATTO $647 \mathrm{~N}$ and various Abberior STAR dyes [14]. ATTO $647 \mathrm{~N}$ and Abberior STAR635 yielded the highest brightness, though the phosphorylated dye Abberior STAR635P had the best signal-to-noise ratio (SNR) due to reduced unspecific background staining. The high brightness of ATTO 647N and contrast of Abberior STAR635P makes them also excellent candidates for cryo-fluorescence microscopy.

In the present paper, we present experimental results for the photon yield of various red dyes at liquid nitrogen temperatures. In particular, we tested the dyes Abberior STAR635P, ATTO 647N, Alexa Fluor 647, ATTO 655, Cy5, and two Silicon-Rhodamine dyes, SiR and (Janelia Farm) JF 646. The intention is to find red dyes that offer the highest localization precision due to their increased photon yield at cryogenic temperatures. 


\section{Results and Discussion}
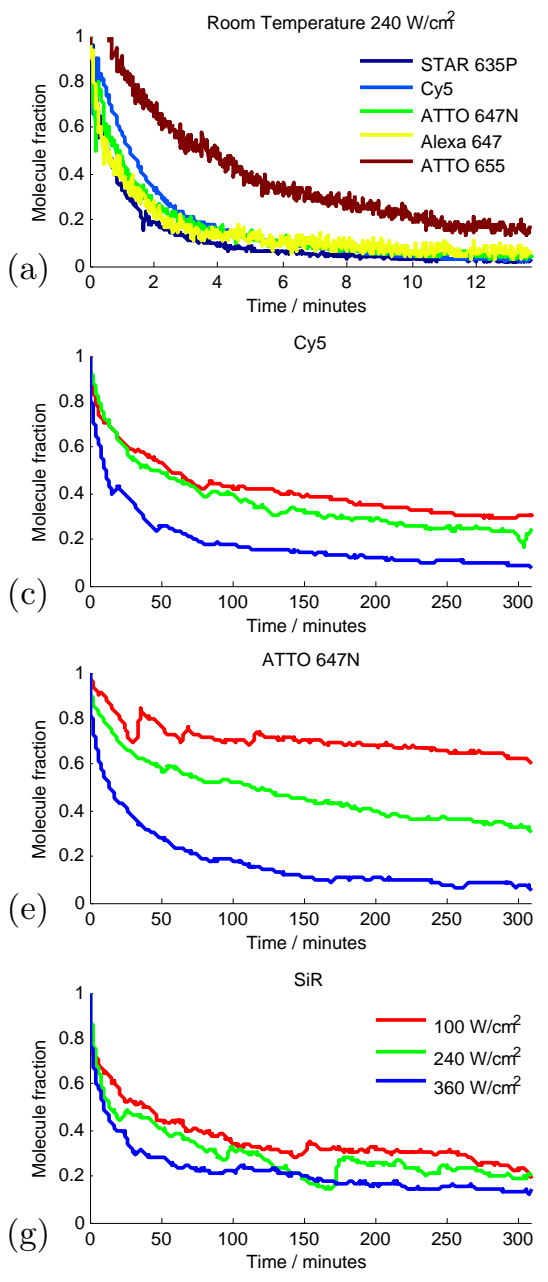
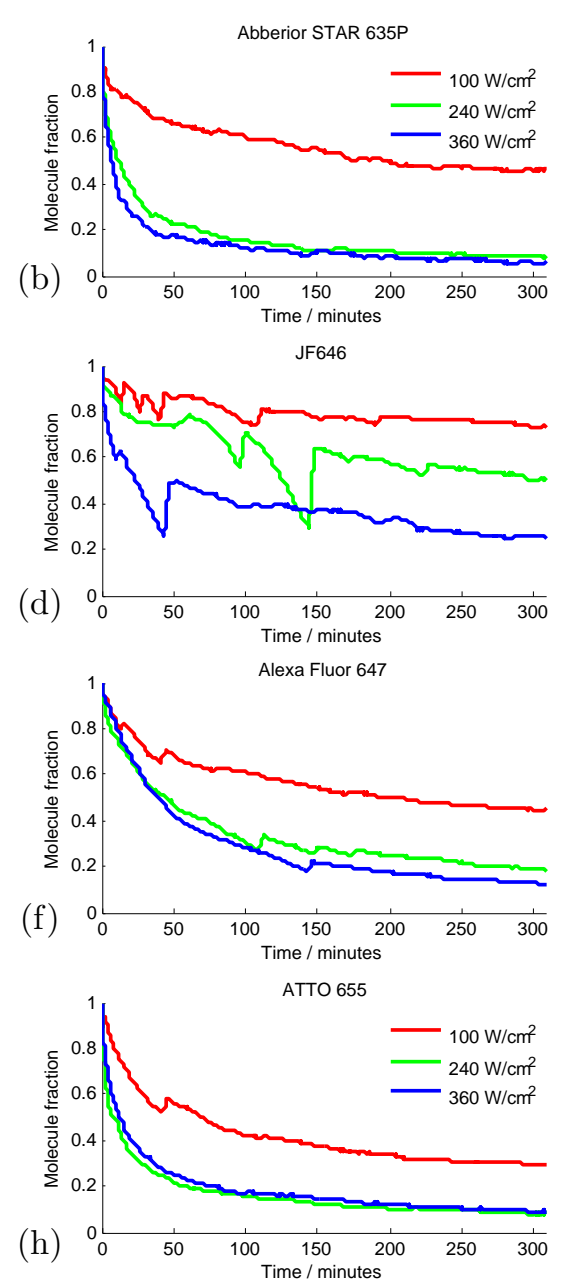

Figure 1: Photobleaching curves at room and cryogenic temperatures, as determined by the number of still fluorescing molecules per frame. (a): Room temperature measurement of Abberior STAR635P, Cy5, ATTO 647N, Alexa Fluor 647 and ATTO 655 at $240 \mathrm{~W} / \mathrm{cm}^{2}$ excitation intensity in the sample plane over 13 minutes. (b)-(h): Decay curves at $\sim 89 \mathrm{~K}$ and 3 different intensities $100 \mathrm{~W} / \mathrm{cm}^{2}$ (red), $240 \mathrm{~W} / \mathrm{cm}^{2}$ (green) and $360 \mathrm{~W} / \mathrm{cm}^{2}$ (blue) for: (b) Abberior STAR635P, (c) Cy5, (d) JF 646, (e) ATTO 647N, (f) Alexa Fluor 647, (g) SiR and (h) ATTO 655. Curves are smoothed with a moving average filter with a span of 200 seconds. The large jumps in the curves are due to axial drift/defocusing, which required manual refocusing while imaging over several hours. Legends for (b)-(h) are identical. 
For estimating the photostability of a dye, we immobilized a sparse concentration of the dye on a quartz surface and determined how many molecules could be localized in an image over the course of time. This number exponentially decayed with increasing time due to photobleaching. Thus, at room temperature we determined values of the half life, the time required to reduce the fluorescing molecules by $50 \%$, ranging from $t_{1 / 2}=36$ seconds for Abberior STAR635P to $t_{1 / 2}=173$ seconds for ATTO 655 (Figure 1a). The excitation intensity for the room temperature measurement was $240 \mathrm{~W} / \mathrm{cm}^{2}$. At cryogenic temperatures of $\sim 89 \mathrm{~K}$, each dye was measured at 3 different intensities, $100 \mathrm{~W} / \mathrm{cm}^{2}, 240$ $\mathrm{W} / \mathrm{cm}^{2}$, and $360 \mathrm{~W} / \mathrm{cm}^{2}$ (Figure 1b-h). The half life was 128 times longer at cryogenic temperatures for ATTO $647 \mathrm{~N}$ at an excitation intensity of $240 \mathrm{~W} / \mathrm{cm}^{2}$ $\left(t_{1 / 2}=111\right.$ minutes compared to $t_{1 / 2}=52$ seconds $)$. The other dyes have a half life at cryogenic temperatures of $t_{1 / 2}=310$ minutes for JF $646, t_{1 / 2}=44$ minutes for Alexa Fluor $647, t_{1 / 2}=43$ minutes for Cy5, $t_{1 / 2}=18$ minutes for SiR, $t_{1 / 2}=12$ minutes for Abberior STAR635P, and $t_{1 / 2}=10$ minutes for ATTO 655 , at the same excitation intensity of $240 \mathrm{~W} / \mathrm{cm}^{2}$. Higher excitation intensities lead to faster bleaching of the fluorescent molecules; reducing the intensity to $100 \mathrm{~W} / \mathrm{cm}^{2}$ decreases the bleaching rate whilst retaining a good signal-tonoise ratio in the images. The slow bleaching of JF 646 , ATTO $647 \mathrm{~N}$, and Abberior STAR635P implies that they would be best for long-lasting cryogenic experiments.

In each image, the number of detected photons from a given molecule can be estimated, and when summing this number over all frames, the total photon yield per molecule till photobleaching is obtained (see Experimental Section for details). The photon yield distribution of ATTO $647 \mathrm{~N}$ at room temperature at $240 \mathrm{~W} / \mathrm{cm}^{2}$ excitation intensity shows an exponential distribution (Figure 2a). Thus, we fitted these photon yield histograms with an exponential decay function, $f(N)=A \cdot \exp (-N / \gamma)$. Before fitting, we excluded the first bin because it can contain falsely identified weakly fluorescent signals. For ATTO $647 \mathrm{~N}$ at room temperature, the mean photon yield from the exponential fit is $\gamma=2.1 \pm 0.1 \cdot 10^{4}$ photons, the uncertainty here is the $95 \%$ confidence interval of the fit. At cryogenic temperatures of $\sim 89 \mathrm{~K}$, the mean photon yield increases by a factor of 240 to $\gamma=5.1 \pm 0.5 \cdot 10^{6}$ photons for ATTO $647 \mathrm{~N}$ at $240 \mathrm{~W} / \mathrm{cm}^{2}$ excitation intensity. The mean photon yields for all the tested dyes at cryogenic temperatures range from $\gamma=2.9 \pm 0.8 \cdot 10^{6}$ for $\mathrm{SiR}$ to $\gamma=6.5 \pm 0.3 \cdot 10^{6}$ for JF 646 at $100 \mathrm{~W} / \mathrm{cm}^{2}$ excitation intensity. This implies a mean theoretical localization precision of around $0.1 \mathrm{~nm}$ using equation 1 with a wavelength of $\lambda=685 \mathrm{~nm}$ and $\mathrm{NA}=0.7$. It is also possible to find the localization precision by calculating the standard deviation of the localizations over a few frames and scaling this by the amount of photons captured. For Abberior star $635 \mathrm{P}$ at $100 \mathrm{~W} / \mathrm{cm}^{2}$ excitation intensity, we found an average standard deviation of $12 \mathrm{~nm}$ over 10 frames for 9 different molecules with an average total photon yield of $7 \cdot 10^{6}$ photons. Scaling with the ratio $\sqrt{N_{10} \text { frames } / N_{\text {total }}}$ gives a mean localization precision of $0.3 \mathrm{~nm}$ which is on the same order of magnitude as the theoretical localization precision. This implies that all tested dyes are suitable for high resolution cryogenic super-resolution microscopy, as this is already smaller than the linker 
length used to attach fluorescent dyes to biological molecules of interest [15].
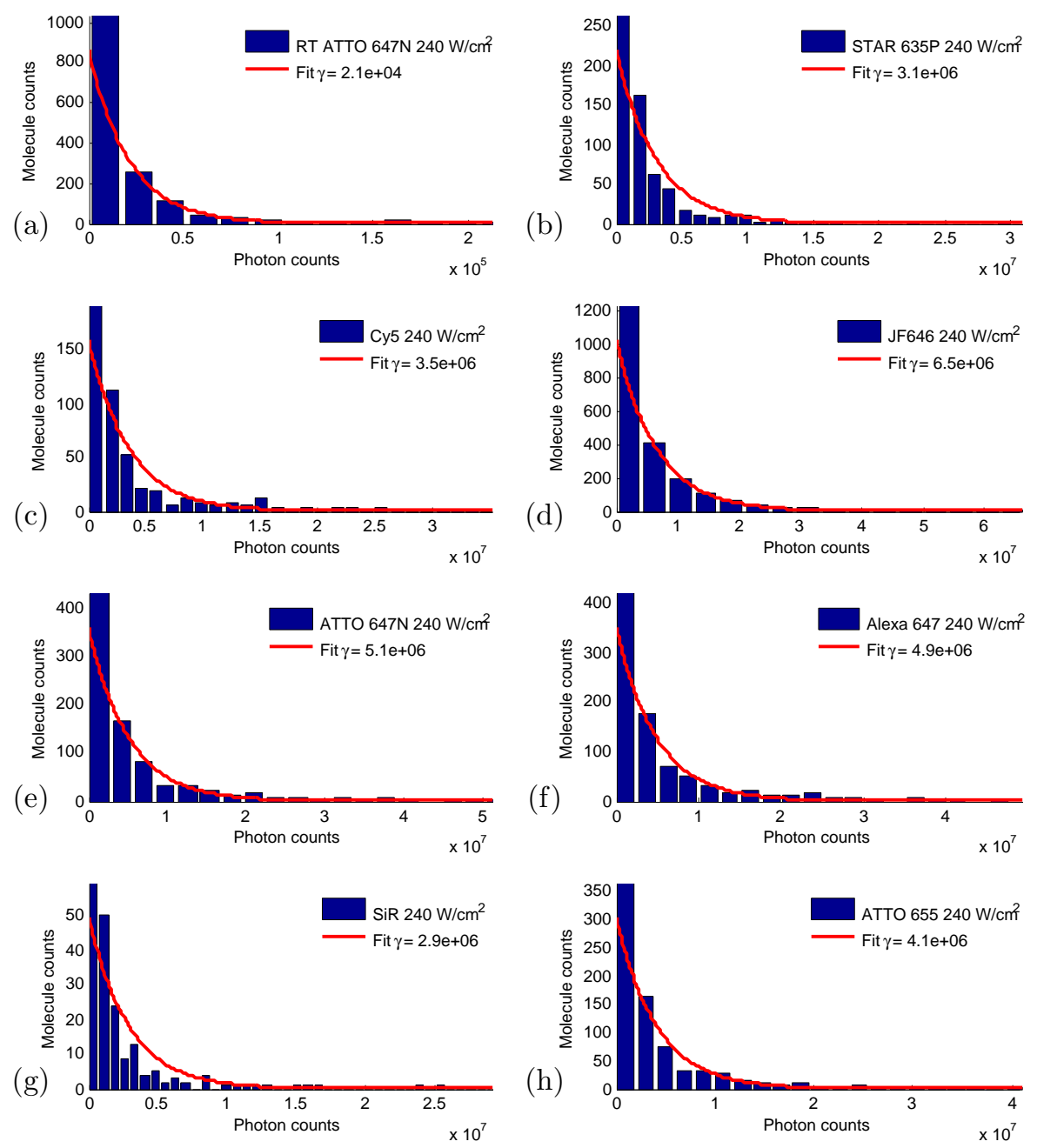

Figure 2: Histogram of photon counts fitted with an exponential decay. (a): Room temperature measurement of ATTO $647 \mathrm{~N}$ at $240 \mathrm{~W} / \mathrm{cm}^{2}$ excitation intensity. Cryogenic measurements $(\sim 89 \mathrm{~K})$ at $240 \mathrm{~W} / \mathrm{cm}^{2}$ excitation intensity for (b) Abberior STAR635P, (c) Cy5, (d) JF 646, (e) ATTO 647N, (f) Alexa Fluor 647, (g) SiR and (h) ATTO 655.

To better compare the results between different dyes, we corrected the measured photon yield numbers by taking into account the different overlap between a dye's emission spectrum and the transmission spectrum of the optical emission filters used in our experiment. Thus, we calculated the photon yield that one 
would observe if the emission filter would perfectly match the emission spectrum of a given dye. Again, we find that in most cases the photon yield $\gamma$ is larger for lower excitation intensities (Figure 3a). The highest determined mean photon yield is $\gamma=11.1 \pm 0.9 \cdot 10^{6}$ photons for the dye Abberior STAR635P at $100 \mathrm{~W} / \mathrm{cm}^{2}$ excitation intensity. The maximum absolute photon yield that we observed (over all detected molecules for a given dye) ranged between $7.9 \cdot 10^{7}$ photons for an SiR dye molecule and $3.6 \cdot 10^{8}$ photons for a JF 646 dye molecule.

A cumulative histogram of emitted photons per molecule at $100 \mathrm{~W} / \mathrm{cm}^{2}$ excitation intensity shows the distribution of detectable photons till photobleaching at cryogenic conditions (Figure $3 \mathrm{~b}$ ). These histograms were normalized to the total molecule count (first left-hand columns in the figure) which is the sum of all molecules that were fluorescent for at least one minute after start of the measurement.

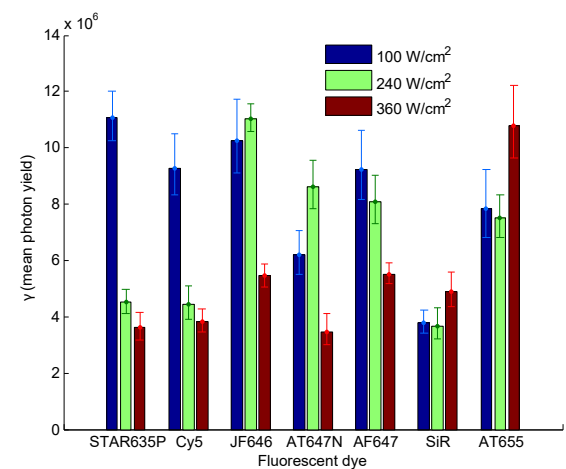

(a)

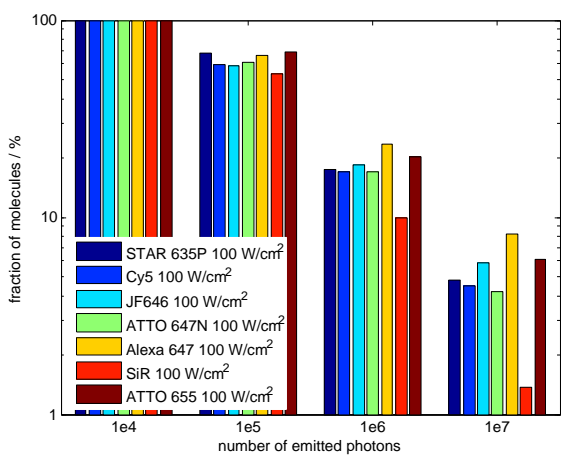

(b)

Figure 3: (a): Mean photon yield $\gamma$ and $95 \%$ confidence interval from the exponential fits of the photostability decay curves of 7 dyes for 3 different intensities at cryogenic temperatures $(\sim 89 \mathrm{~K})$. (b): Cumulative histogram of the number of emitted photons per molecule at $100 \mathrm{~W} / \mathrm{cm}^{2}$ excitation intensity and $\sim 89 \mathrm{~K}$. All molecule numbers were referenced against the number of molecules that survived the first minute of measurement time ( $100 \%$, left columns in bar plot).

Finally, we can use equation 1 to calculate the minimal required photon yield per molecule to achieve a localisation precision of $1 \mathrm{~nm}$, which is $6 \cdot 10^{4}$ photons for our system. Typically, $80 \%$ of all molecules emit $6 \cdot 10^{4}$ photons or more for the tested dyes and different excitation intensities (Figure 4a). The chance of finding a molecule with a localisation precision equal to or better than 0.1 $\mathrm{nm}\left(\geq 6 \cdot 10^{6}\right.$ photons) was found to be higher for a lower excitation intensity $\left(100 \mathrm{~W} / \mathrm{cm}^{2}\right)$ for all dyes except ATTO 655 (Figure $4 \mathrm{~b}$ ). The dyes with the highest probability of achieving $\leq 0.1 \mathrm{~nm}$ localisation precision are Alexa Fluor $647(11.1 \%)$, ATTO 655 (14.4\%), and JF 646 (8.7\%). The molecules emitting these amounts of photons were fluorescing during almost the entire experiment suggesting that the photon count from these molecules could be futher increased 
by extending the measurement time.

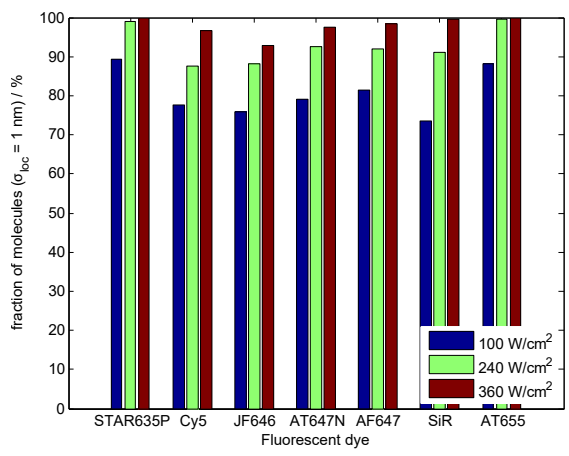

(a)

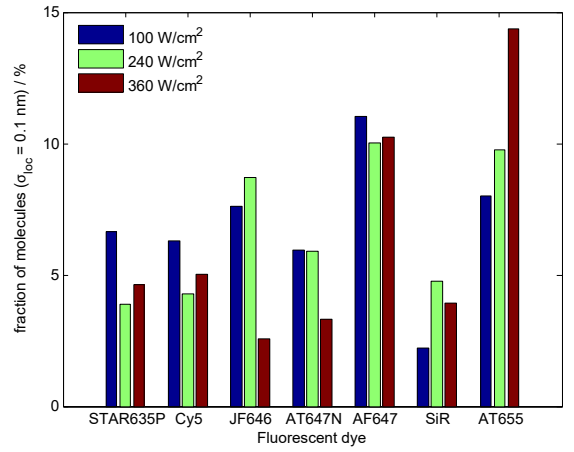

(b)

Figure 4: Fraction of molecules achieving a certain theoretical localisation precision for 7 different dyes and 3 excitation intensities at cryogenic temperatures ( $\sim 89 \mathrm{~K}) .(\mathbf{a}): \sim 1 \mathrm{~nm}\left(6 \cdot 10^{4}\right.$ photons $)$ (b): $\sim 0.1 \mathrm{~nm}\left(6 \cdot 10^{6}\right.$ photons $)$.

\section{Conclusions}

The mean photon yield of ATTO $647 \mathrm{~N}$ (uncorrected for spectra) of $5 \cdot 10^{6}$ photons at $240 \mathrm{~W} / \mathrm{cm}^{2}$ and $3 \cdot 10^{6}$ photons at $360 \mathrm{~W} / \mathrm{cm}^{2}$ agrees well with the result found by $\mathrm{Li}$ et al. $\left(3.5 \cdot 10^{6}\right.$ photons at $\left.300 \mathrm{~W} / \mathrm{cm}^{2}\right)$ [7]. The maximal photon yield at liquid nitrogen temperatures is lower than at liquid helium temperatures, with only $\sim 5 \%$ emitting $10^{7}$ photons or more compared to $>20 \%$ at liquid helium temperatures as found by Weisenburger et al. [16]. Although the photon yield is lower, liquid nitrogen is more suitable for fluorescence microscopy as phonons are frozen out at liquid helium temperatures leading to a significant narrowing of the excitation and emission lines [17], making efficient excitation of a fluorophore difficult.

All tested dyes show good performance for cryogenic localization, achieving an average theoretical localization precision around $0.1 \mathrm{~nm}$ from $3.5 \cdot 10^{6}$ to $11 \cdot 10^{6}$ photons. This is a more than hundred-fold increase in photon yield over room temperature, leading to a 10 fold increase in localization precision. From this study it seems that JF 646, Alexa Fluor 647, and ATTO 655 give the highest photon yields resulting in the best localization precision. It is assumed that due to the low density, most identified molecules are indeed single-molecules, which is also corroborated by the observed single-step bleaching of almost all identified molecules.

At $\sim 89 \mathrm{~K}$ we found that the mean photon yield of the best dye was 3.1 times higher than the dye with the lowest mean photon yield. This means that there is a difference factor of $1.8(=\sqrt{3.1})$ between the localization precision of the tested dyes. However, photon yield at cryogenic temperatures is not the only factor to be taken into consideration for dye selection. Other important 
factors are ease of biological labeling, solubility, cell permeability, net charge, extinction coefficient, stability, and non-specific staining.

This study has only addressed photon yield and its impact on localization of single molecules. To perform super-resolution measurements on biological samples, it is still necessary to induce sparsity so that individual molecules can be localized. One obvious way to do that could be to use PALM or dSTORM. All the dyes that were studied here do not show significant photoswitching which could be used for dSTORM. Also, we did not see any photoactivation of PA-JF 646 [18] at cryogenic conditions, although this dye shows excellent photoactivation at room temperature. Thus, besides for maximum photon yield, future work has also to screen for photoswitching ability at cryogenic temperatures. Besides organic dyes, promising candidates might be fluorescent proteins. For example, it is known that the fluorescent protein PA-GFP can be activated under cryogenic conditions [9], indicating that cryo-PALM may be feasible using this protein. Another method could be to induce sparsity by using polarizationdependent excitation and stimulated emission depletion [19, 20], although this will require a much more demanding experimental setup. 


\section{Experimental Section}

\subsection{Cryostat}

The microscope used in this study is built solely for cryogenic Liquid Nitrogen (LN2) temperature measurements and has already been extensively described earlier [7]. Samples coated on $200 \mu \mathrm{m}$ thick quartz cover slips are mounted in the cryostat and are imaged through a $0.5 \mathrm{~mm}$ thick quartz window to maintain vacuum. Quartz is used instead of glass due to its reduced autofluorescence. The sample chamber is pumped to a pressure $\leq 1.6 \cdot 10^{-5}$ mbar. The cryostat is filled with LN2 and the temperature is left to stabilize for one hour. Once stabilized, it is possible to measure for more than 5 hours with a temperature between $89-93 \mathrm{~K}$ and a typical temperature change of $0.1 \mathrm{~K} /$ hour.

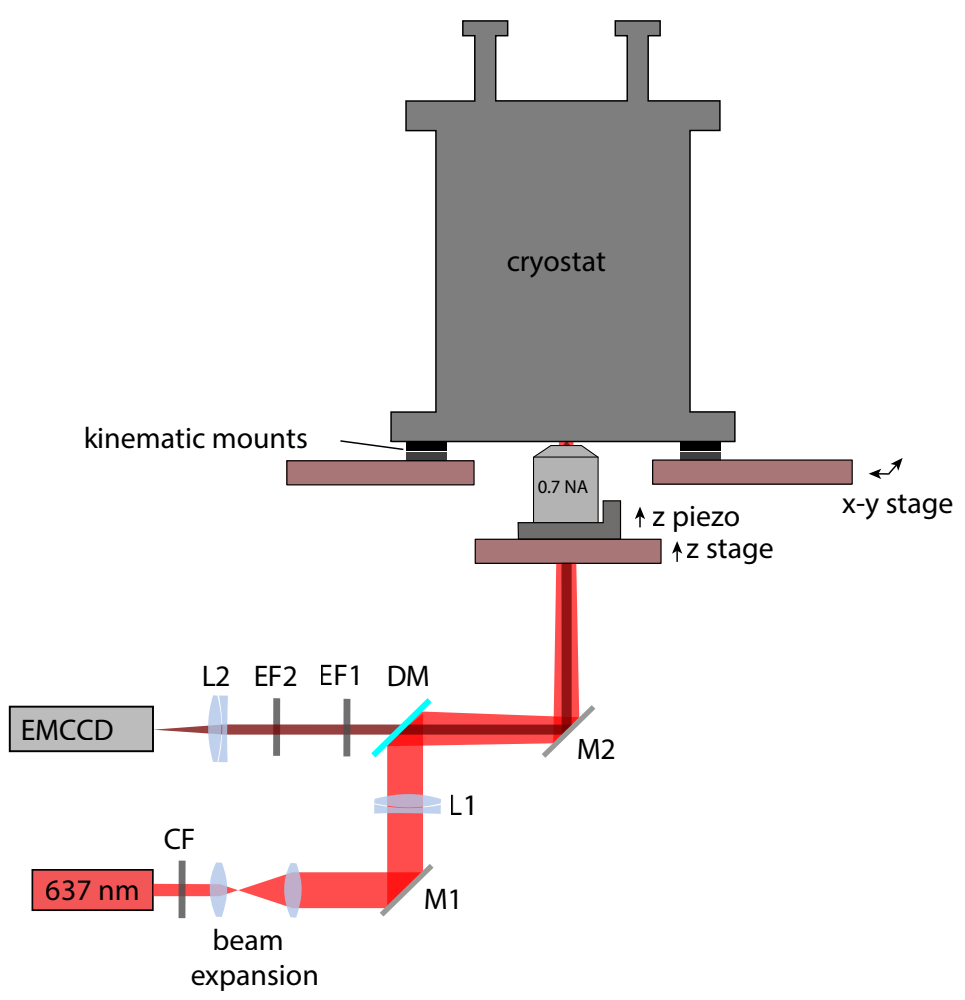

Figure 5: Cryo-fluorescence microscope with the sample mounted in the vacuum environment of the cryostat. Imaging is done with a long working distance 0.7NA objective onto an EMCCD camera. The excitation laser is focused onto the back focal plane of the objective and illuminates the field of view of the camera. 


\subsection{Fluorescence microscope}

The whole cryostat is mounted on a motorized $\mathrm{x}-\mathrm{y}$ stage, and the objective (LUCPLFLN 60x/0.7NA, Olympus) is mounted on both a motorized z stage and a piezo z-stage (PIFOC, PI). The excitation and emission path is that of a typical epi-fluorescence microscope (Figure 5). The red $637 \mathrm{~nm}$ continuous wave excitation laser (OEM-SD-637-500, CNI) is filtered by a clean-up filter CF (HC $640 / 14$, Semrock), expanded by a telescope (Plano convex $\mathrm{f}=30 \mathrm{~mm}, \mathrm{f}=100 \mathrm{~mm}$, Thorlabs), and focused onto the back focal plane of the objective by lens L1. The dichroic mirror DM (Di01-R405/488/532/635, Semrock) reflects the excitation laser and transmits the fluorescence from the sample. The fluorescence is filtered by two emission filters EF1 (FF01-446/510/581/703, Semrock) and EF2 (ET $685 / 50$, Chroma) and focused by lens L2 (AC508-180-A-ML, Thorlabs) onto an EMCCD camera (Ixon Ultra 897, Andor). Excitation intensities are estimated by measuring the power of the parallel beam after the objective with a power meter and dividing it by the illuminated area in the sample plane.

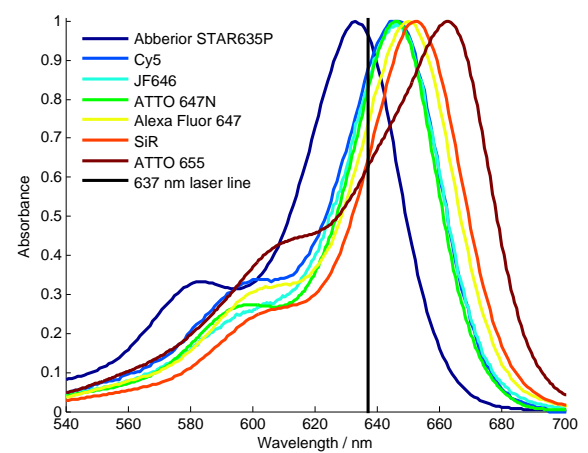

(a) Absorption spectra

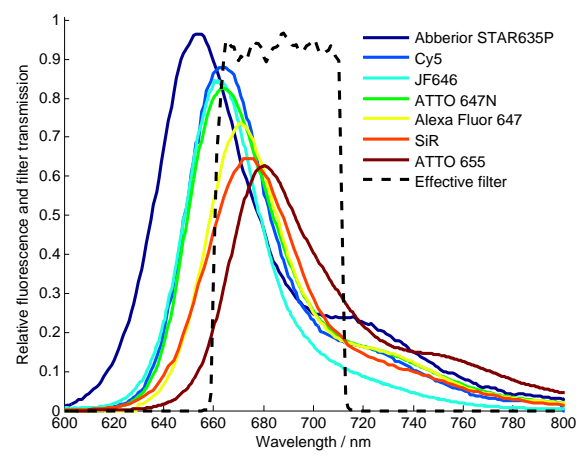

(b) Effective emission spectra

Figure 6: Absorption and emission spectra of Abberior STAR635P, Cy5, JF 646, ATTO 647N, Alexa Fluor 647, SiR and ATTO 655. The emission spectra are normalized with excitation at $637 \mathrm{~nm}$. The effective filter is the combined action of the dichroic mirror, quad-band filter and ET685/50 bandpass filter.

We investigated the dyes; Abberior STAR635P, Cy5, JF 646 (spectrum from [21]), ATTO 647N, Alexa Fluor 647, SiR and ATTO 655, which have very similar excitation and emission spectra (figure 6). The emission spectra are scaled to their values at $637 \mathrm{~nm}$ excitation, and they are plotted along with the transmission curve of the emission filter combination used in our experiment (figure $6 \mathrm{~b})$. To correct for the effect of the emission filter and imperfect excitation wavelength, we up-scaled photon count numbers by the scaling factor

$$
\text { scaling factor }=\frac{\int_{\mathrm{abs}_{\text {peak }}+10 \mathrm{~nm}}^{800 \mathrm{~nm}}(\text { em. spectrum }) d \lambda}{\int_{600 \mathrm{~nm}}^{800 \mathrm{~nm}}(\text { scaled em. spectrum }) \cdot(\text { em. filter function }) d \lambda} .
$$


In the denominator, we start the integration only $10 \mathrm{~nm}$ after the absorption peak to model the gradual transition between reflection and transmission of a dichroic mirror. In order to obtain the compensated photon counts, the raw photon count number is multiplied by this scaling factor for each dye. The scaling factor ranges from 1.7 for JF 646 to 2.5 for Abberior STAR635P.

\subsection{Sample preparation}

Initially, each organic dye is diluted with distilled, de-ionized and filtered water to a $100 \mathrm{pM}$ concentration. $5 \mu \mathrm{L}$ of this dilution is deposited on a quartz slide and spin-coated at 3000 RPM for 45 seconds. The quartz slide is imaged at room temperature to determine the density of molecules in the $136.5 \mu \mathrm{m} \times$ $136.5 \mu \mathrm{m}$ field of view. We aimed at having $\sim 400$ single molecules in the field of view to balance sparsity whilst retaining enough molecules after extensive bleaching. Dye concentrations were adjusted between $25 \mathrm{pM}$ and $500 \mathrm{pM}$ to achieve the desired number of initial molecules. At cryogenic temperatures, the initial amount of single molecules was found to be higher, 400-1200 instead, because at room temperature many molecules would have already been bleached during focusing, thus lowering the amount of molecules. Concentrations were not compensated for this effect but kept at 200-400 localizations at room temperature to maintain consistency between measurements.

\subsection{Image acquisition}

For each cryogenic photon yield measurement, the full time duration over which the cryostat is stable is used, to maximize the total amount of captured photons. In total, 18,000 frames with 1 second exposure time are recorded, resulting in 130-220 photons per molecule per frame. Each frame has a readout time of $25 \mathrm{~ms}$, resulting in a total measurement of $\sim 5$ hours. All data is acquired with an EM gain of 100, and an EMCCD sensor temperature of $-45^{\circ} \mathrm{C}$. Although the system is very stable, there is still a typical axial drift of $3 \mu \mathrm{m}$ over 5 hours causing defocusing. To compensate for this, it is necessary to manually refocus the system periodically. We found that refocusing every 10 minutes during the first hour and then after every hour thereafter is sufficient.

\subsection{Image analysis}

The Analog-Digital Units (ADU) of the camera images can be converted into photons when knowing the bias $b$, EM gain $G$, and specific sensitivity $s$ of the camera, by using the equation

$$
N=(A-b) \frac{s}{G} .
$$

The amplitude of the Gaussian fit to a single molecule image was used to calculate the total number of emitted photons from a molecule in each frame. 
Recently, it has been shown that 2D Gaussian fitting can lead to a 10-30\% underestimation of the photon counts [22], but we did not take this into account here. After tracking each molecule over the course of the whole measurement, the total number of emitted photons per molecule was determined.

Molecule tracking was done using TrackNTrace, an open-source MATLAB framework for localizing and tracking molecules [23]. TrackNTrace identifies molecules in two steps. We used a cross-correlation for the candidate detection step, and a 2D Gaussian fitting for the refinement step. The localized molecules were tracked using a nearest neighbor approach.

The tracking procedure was applied twice, where the initial tracking is done with no frame gaps, meaning that blinking leads to new tracks. This results in reliable data to calculate the average drift from frame to frame. The data is then adjusted for this average drift and tracked again, but now frame gaps are allowed (up to the total measurement duration). This allows molecules to blink but still be identified as one and the same molecule. This method reduces the chance of identifying distinct molecules as a single molecule. Combined with the low density of molecules, we assume that almost all identified molecules are single.

\section{Acknowledgments}

We thank Luke Lavis (HHMI, Janelia Farm) for providing us with the PAJF 646 and JF 646 dyes. B.R. and C.N.H. acknowledge European Research Council grant no. 648580. W.L., I.G. and J.E. acknowledge financial support by the Deutsche Forschungsgemeinschaft (DFG, project A06 of the SFB 860).

\section{Keywords}

single-molecule photon yield, cryo-fluorescence microscopy, single-molecule localization precision, super-resolution microscopy

\section{References}

[1] S.W. Hell. Microscopy and its focal switch. Nature Methods, 6(1):24-32, 2009.

[2] E. Betzig, G.H. Patterson, R. Sougrat, O.W. Lindwasser, S. Olenych, J.S. Bonifacino, M.W. Davidson, J. Lippincott-Schwartz, and H.F. Hess. Imaging intracellular fluorescent proteins at nanometer resolution. Science, 313(5793):1642-5, 2006.

[3] M.J. Rust, M. Bates, and X.W. Zhuang. Sub-diffraction-limit imaging by stochastic optical reconstruction microscopy (STORM). Nature Methods, 3(10):793-795, 2006. 
[4] S. Stallinga and B. Rieger. Accuracy of the Gaussian Point Spread Function model in 2D localization microscopy. Optics Express, 18(24):24461-24476, 2010.

[5] B. Rieger and S. Stallinga. The lateral and axial localization uncertainty in super-resolution light microscopy. ChemPhysChem, 15(4):664-670, 2014.

[6] S. Weisenburger, B. Jing, A. Renn, and V. Sandoghdar. Cryogenic localization of single molecules with Angstrom precision. SPIE NanoScience + Engineering, 8815:88150D, 2013.

[7] W. Li, S.C. Stein, I. Gregor, and J. Enderlein. Ultra-stable and versatile widefield cryo-fluorescence microscope for single-molecule localization with sub-nanometer accuracy. Optics Express, 23(3):3770-83, 2015.

[8] R. Kaufmann, C. Hagen, and K. Grünewald. Fluorescence cryo-microscopy: Current challenges and prospects. Current Opinion in Chemical Biology, 20(1):86-91, 2014.

[9] Y.-W. Chang, S. Chen, E.I. Tocheva, A. Treuner-Lange, S. Löbach, L. Søgaard-Andersen, and G.J. Jensen. Correlated cryogenic photoactivated localization microscopy and cryo-electron tomography. Nature Methods, 11(7):737-739, 2014.

[10] R.P.J. Nieuwenhuizen, K.A. Lidke, M. Bates, D.L. Puig, D. Grünwald, S. Stallinga, and B. Rieger. Measuring image resolution in optical nanoscopy. Nature Methods, 10(6):557-562, 2013.

[11] A. Löschberger, S. van de Linde, M.-C. Dabauvalle, B. Rieger, M. Heilemann, G. Krohne, and M. Sauer. Super-resolution imaging visualizes the eightfold symmetry of gp210 proteins around the nuclear pore complex and resolves the central channel with nanometer resolution. Journal of Cell Science, 125(3):570-575, 2012.

[12] J. Broeken, H. Johnson, D.S. Lidke, S. Liu, R.P.J. Nieuwenhuizen, S. Stallinga, K.A. Lidke, and B. Rieger. Resolution improvement by 3D particle averaging in localization microscopy. Methods and Applications in Fluorescence, 3(1), 2015.

[13] J.E. Berlier, A. Rothe, G. Buller, and J. Bradford. Quantitative comparison of long-wavelength Alexa Fluor dyes to Cy dyes: fluorescence of the dyes and their bioconjugates. The Journal of Histochemistry \&3 Cytochemistry, 51(12):1699-1712, 2003.

[14] C.A. Wurm, K. Kolmakov, F. Göttfert, H. Ta, M. Bossi, H. Schill, S. Berning, S. Jakobs, G. Donnert, V.N. Belov, and S.W. Hell. Novel red fluorophores with superior performance in STED microscopy. Optical Nanoscopy, $1(1): 7,2012$. 
[15] S. Weisenburger, D. Boening, B. Schomburg, K. Giller, S. Becker, C. Griesinger, and V. Sandoghdar. Cryogenic optical localization provides 3D protein structure data with Angstrom resolution. Nature Methods, 14(2), 2017.

[16] S. Weisenburger, B. Jing, D. Hänni, L. Reymond, B. Schuler, A. Renn, and V. Sandoghdar. Cryogenic colocalization microscopy for nanometerdistance measurements. ChemPhysChem, 15(4):763-770, 2014.

[17] W.E. Moerner and M. Orrit. "Illuminating single molecule in condensed matter". Science, 283(5408):1670, 1999.

[18] J.B. Grimm, B.P. English, H. Choi, A.K. Muthusamy, B.P. Mehl, P. Dong, T.A. Brown, J. Lippincott-Schwartz, Z. Liu, T. Lionnet, and L.D. Lavis. Bright photoactivatable fluorophores for single-molecule imaging. Nature Methods, 13(12):985-988, 2016.

[19] N. Hafi, M. Grunwald, L.S. van den Heuvel, T. Aspelmeier, J.-H. Chen, M. Zagrebelsky, O.M. Schütte, C. Steinem, M. Korte, A Munk, and P.J. Walla. Fluorescence nanoscopy by polarization modulation and polarization angle narrowing. Nature methods, 11(5):579-84, 2014.

[20] C.N. Hulleman, M. Huisman, R.J. Moerland, D. Grünwald, S. Stallinga, and B. Rieger. Fluorescence polarization control for on-off switching of single molecules at cryogenic temperatures. under review.

[21] J.B. Grimm, A.K. Muthusamy, Y. Liang, T.A. Brown, W.C. Lemon, R. Patel, R. Lu, J.J. Macklin, P.J. Keller, N. Ji, and L.D. Lavis. A general method to fine-tune fluorophores for live-cell and in vivo imaging. Nature Methods, 14(10):987-994, 2017.

[22] R.Ø. Thorsen, C.N. Hulleman, S. Stallinga, and B. Rieger. Signal photon count estimation in single molecule localization microscopy. In Quantitative BioImaging Conference (QBI), Göttingen, Germany, January 4-6, 2018.

[23] S.C. Stein and J. Thiart. TrackNTrace: A simple and extendable opensource framework for developing single-molecule localization and tracking algorithms. Scientific Reports, 6:37947, 2016. 